\title{
The Cost-Effectiveness of Alogliptin Versus Sulfonylurea as Add-on Therapy to Metformin in Patients with Uncontrolled Type 2 Diabetes Mellitus
}

\author{
Jason Gordon $\cdot$ Phil McEwan $\cdot$ Michael Hurst $\cdot$ Jorge Puelles
}

Received: August 23, 2016 / Published online: October 27, 2016

(C) The Author(s) 2016. This article is published with open access at Springerlink.com

\begin{abstract}
Introduction: ENDURE (ClinicalTrials.gov identifier, NCT00856284), a multicenter, double-blind, active-controlled study of 2639 patients with uncontrolled type 2 diabetes mellitus (T2DM), found that metformin in
\end{abstract}

Enhanced content To view enhanced content for this article go to http://www.medengine.com/Redeem/ A017F060797F273D.

Electronic supplementary material The online version of this article (doi:10.1007/s13300-016-0206-7) contains supplementary material, which is available to authorized users.

J. Gordon $(\bowtie) \cdot$ P. McEwan · M. Hurst

Health Economics and Outcomes Research Ltd, Cardiff, UK

e-mail: jason.gordon@heor.co.uk

J. Gordon

School of Medicine, University of Nottingham,

Nottingham, UK

J. Gordon

Department of Public Health, University of

Adelaide, Adelaide, Australia

\section{P. McEwan}

Swansea Centre for Health Economics, Swansea

University, Swansea, UK

J. Puelles

Global Outcomes Research, Takeda Development

Centre Europe Ltd, London, UK combination with alogliptin (12.5 and $25 \mathrm{mg}$ doses), when compared to standard add-on therapy (sulfonylurea, SU), exerted sustained antihyperglycemic effects over 2 years. This economic analysis of ENDURE aimed to quantify the relationship between increased glycemic durability and cost-effectiveness of alogliptin in the UK clinical setting, and communicate its sustained glycemic benefit in economic terms.

Methods: Using baseline characteristics and treatment effects from the ENDURE trial population, between-group cost-effectiveness analyses compared the combined use of metformin and alogliptin (MET $\left.+\mathrm{ALO}_{12.5 / 25}\right)$ in patients with inadequately controlled T2DM, as an alternative to metformin and SU $(\mathrm{MET}+\mathrm{SU})$. In scenario analyses, an intragroup cost-effectiveness analysis compared $\mathrm{MET}+\mathrm{ALO}_{12.5 / 25}$ with $\mathrm{MET}+\mathrm{SU} ; \quad \mathrm{a}$ between-group cost-effectiveness analysis also compared $\mathrm{MET}+\mathrm{ALO}_{12.5 / 25}$ versus $\mathrm{MET}+\mathrm{SU}$ within a subpopulation of patients who achieved HbA1c control $(<7.5 \%)$ at 2 years on study drug.

Results: Compared with baseline profiles of patients, combination therapies with 
alogliptin or SU were associated with improvements in length and quality of life and were cost-effective at established norms. Despite increased drug acquisition costs, alogliptin at $12.5 \mathrm{mg}$ and $25 \mathrm{mg}$ doses resulted in greater predicted lifetime quality-adjusted life year (QALY) gains with associated incremental cost-effectiveness ratios (ICERs) of $£ 10,959 / \mathrm{QALY}$ and $£ 7217 / \mathrm{QALY}$ compared to $\mathrm{SU}$, respectively.

Conclusion: The ENDURE trial and the present cost-effectiveness analysis found that the glycemic durability of alogliptin therapy was associated with improved long-term patient outcomes, QALY gains, and ICERs that were cost-effective when evaluated against standard threshold values. Alogliptin therefore represents a cost-effective treatment alternative to SU as add-on therapy to metformin in patients with poorly managed T2DM.

Funding: Takeda Development Centre Europe Ltd.

Keywords: Alogliptin; Cost-effectiveness analysis; Glycemic durability; Sulfonylurea; Second-line therapy; Type 2 diabetes mellitus

\section{INTRODUCTION}

Type 2 diabetes mellitus (T2DM) is a chronic metabolic disorder that imposes major social and economic burdens on public health in the UK. In addition to the direct healthcare costs associated with managing T2DM, the societal and productivity consequences of T2DM also incur significant indirect costs. In 2010-11, it was estimated that T2DM affected 3.4 million adults in the UK, the cost of which exceeded $£ 21.7$ billion [1]. This was made up of $£ 13$ billion in indirect expenses and $£ 8.7$ billion in direct healthcare costs [1], which account for up to $10 \%$ of all NHS expenditure [2]. Economic projections have hypothesized that the prevalence of T2DM in the UK will rise to 5.6 million adults in 2035-36, and will incur direct NHS costs exceeding $£ 15.1$ billion. The indirect costs of the morbidity and mortality associated with T2DM were also projected to rise, to $£ 20.5$ billion by 2035-36 [1].

More than $75 \%$ of NHS expenditure on T2DM is related to the costs of treating the complications of $\mathrm{T} 2 \mathrm{DM}$, rather than the ongoing management of the condition itself [1]. These complications include cardiovascular events, neuropathy, renal disorders, visual impairment, and amputations, many of which are avoidable consequences of suboptimal glycemic control. The National Institute for Health and Care Excellence (NICE) recommends that $\mathrm{T} 2 \mathrm{DM}$ therapy ought to lower glycated hemoglobin A1c (HbA1c) levels to $48 \mathrm{mmol} / \mathrm{mol} \quad(6.5 \%) \quad$ [2]; however, approximately $30 \%$ of patients fail to reach and maintain this goal [3]. Effective management of T2DM, through improvements to current treatment strategies, has the potential to reduce adverse micro- and macrovascular complications, and their associated burden.

Clinical guidelines for the management of T2DM initially advocate metformin, in combination with diet and lifestyle changes $[2,4]$. However, given the progressive nature of T2DM due to declining beta cell function, long-term glycemic management is invariably associated with the requirement for therapy escalation [5, 6]. In patients suboptimally controlled on metformin monotherapy, sulfonylurea (SU) is a common second-line treatment option [2]. Whilst combination therapy with drugs of this class is associated with significant reductions in $\mathrm{HbA1c}$, SUs are 
additionally associated with weight gain and an increased risk of hypoglycemia [7]. The risk of these adverse events is further exacerbated when secondary treatment failure progressively necessitates the addition of further oral therapies and/or insulin initiation [6].

Inhibitors of dipeptidyl peptidase-4 (DPP-4) exert antihyperglycemic effects in T2DM, without increased risk of weight gain or hypoglycemic events [7]. Alogliptin is a selective inhibitor of DPP-4, and its antidiabetic efficacy in monotherapy and combination therapy has been established in clinical studies over 1 year [8-10]. To investigate the long-term glycemic durability of alogliptin, ENDURE (Efficacy and safety of alogliptin plus metformin compared to glipizide plus metformin in subjects with type 2 diabetes mellitus; ClinicalTrials.gov identifier, NCT00856284) was a multicenter, double-blind, active-controlled trial that compared alogliptin with an SU (glipizide) in combination with metformin in poorly managed T2DM over 2 years [11]. The trial found that, in patients with inadequate glycemic control following stable-dose metformin treatment, combination therapy with alogliptin (12.5 and $25 \mathrm{mg}$ once daily) was associated with significant improvements in HbA1c, fasting plasma glucose, and the incidence of weight gain, hyperglycemic rescue, and hypoglycemic events over 2 years [11]. In a post hoc analysis of ENDURE, a significantly greater proportion of the alogliptin cohort achieved the composite endpoint of glycemic control, without weight gain or hypoglycemia [12].

Although ENDURE demonstrated the clinical effectiveness of alogliptin compared to SU as a second-line therapy for T2DM, further studies are required to determine whether its durability benefits may translate to improved cost-effectiveness. The present economic analysis of ENDURE aimed to evaluate the relative cost-effectiveness of alogliptin compared to SU in the UK clinical setting.

\section{METHODS}

\section{Patient Population}

Baseline characteristics and treatment effects were sourced from the ENDURE trial population $(N=2639)$ [11]. Patients were randomized to receive $12.5 \mathrm{mg}$ alogliptin once daily $(n=880)$, $25 \mathrm{mg}$ alogliptin once daily $(n=885)$, or $5 \mathrm{mg}$ glipizide once daily ( $n=874)$ for 104 weeks, in combination with at least $1500 \mathrm{mg}$ open-label metformin once daily (or maximum tolerated dose). The model cohort was considered to be representative of UK patients who would be eligible to receive alogliptin as part of a UK treatment strategy.

\section{Cost-Effectiveness Model}

Economic analysis of ENDURE trial data was performed using the widely published and validated IMS Core Diabetes Model (CDM), a generic (non-product-specific) computer simulation model used to predict the long-term incidence of adverse events and the economic consequences of interventions in the management of T2DM [13].

The CDM is a fixed-time increment stochastic model comprised of 17 interrelated state transition Markov submodels, with each submodel using time, state, and patient-dependent probabilities. Monte Carlo simulations are performed at the individual patient level using tracker variables to accommodate complex interactions between individual complication submodules. The CDM simulates diabetes-related micro- and 
macrovascular complications (angina, myocardial infarction, congestive heart failure, stroke, peripheral vascular disease, diabetic retinopathy, macular edema, cataract, hypoglycemia [nocturnal, severe, and symptomatic], ketoacidosis, lactic acidosis, nephropathy and end-stage renal disease, neuropathy, foot ulcer, and amputation), cardiovascular, and non-specific mortality. It accommodates direct and indirect costs, adjusts for quality of life, and performs cost-effectiveness and cost-utility analyses. Separate transition probabilities and management strategies for type 1 and type 2 diabetes are utilized within the model, and source data for model parameters are obtained from a broad range of published clinical and epidemiological studies, predominantly the Diabetes Control and Complications Trial (DCCT) [14] and Framingham studies [15] for type 1 diabetes and UKPDS studies [16, 17] for T2DM.

Patient progression through the model is determined by baseline clinical and demographic characteristics. The progression of $\mathrm{T} 2 \mathrm{DM}$ is modelled using annual time increments. As the simulation progresses, time-dependent risk factors are updated or modified according to a therapy change, thereby altering the likelihood of event occurrence.

\section{Analyses}

Within each analysis, a cohort of 1000 patients was simulated for each treatment arm based on the baseline profile and treatment effect adjusted for distributions in the deviation of CDM inputs. Each patient was simulated for a lifetime time horizon (excluding where model inputs were fluctuating as part of a sensitivity analysis) up to a maximum of 50 years on a yearly cycle. Discount rates for both cost and utilities were set to $3.5 \%$.

Costs were adjusted for inflation (where necessary), set against 2015 using the hospital and community health services (HCHS) index compiled by the Personal Social Services Research Unit (PSSRU) [18]. The annual cost of each regimen was input into the $\mathrm{CDM}$ as an annual cost encompassing both the treatment and consumables (test strips, lancets, and needles) required to administer and manage the treatment. The treatment and consumables were calculated using both the daily cost obtained from the latest Monthly Index of Medical Specialties (MIMS) [19] and daily usage guidelines for all individual drug regimens and consumables obtained from either the ENDURE study protocol [11] or daily usage guidelines from NICE [2]. Where relevant, an average cost of all relevant products was applied unless explicitly defined within the treatment arm (including metformin, alogliptin, and glipizide). Additional complication specific costs and overall utility consequences were applied on a per cycle basis based on the predicted occurrence of diabetes-related complications. All utilities and disutility rates were sourced from relevant literature of patients with T2DM (see appendix in the Supplementary Material). Modelled costs and utilities are provided in Tables S1-S8 in the Supplementary Material.

Across all analyses, CDM input data for the baseline cohort profile and treatment effect were sourced from published trial data [11] supplemented with validated patient level ENDURE data where required. The baseline profiles used are presented in Table 1; the treatment effects for both the overall population and subpopulation of patients with HbA1c less than $7.5 \%$ at week 104 that were input into the CDM are presented in Tables 2 and 3 , respectively. 


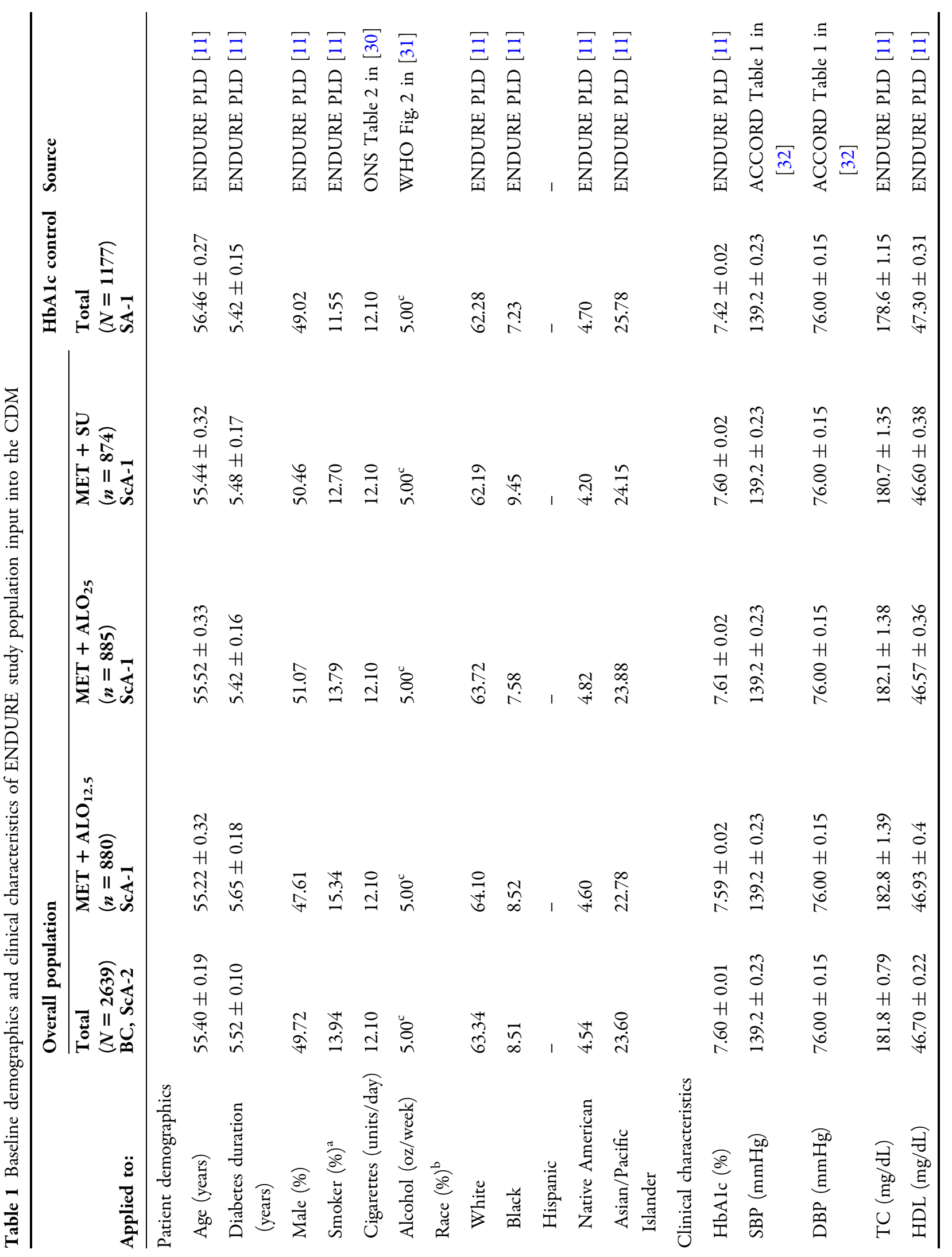




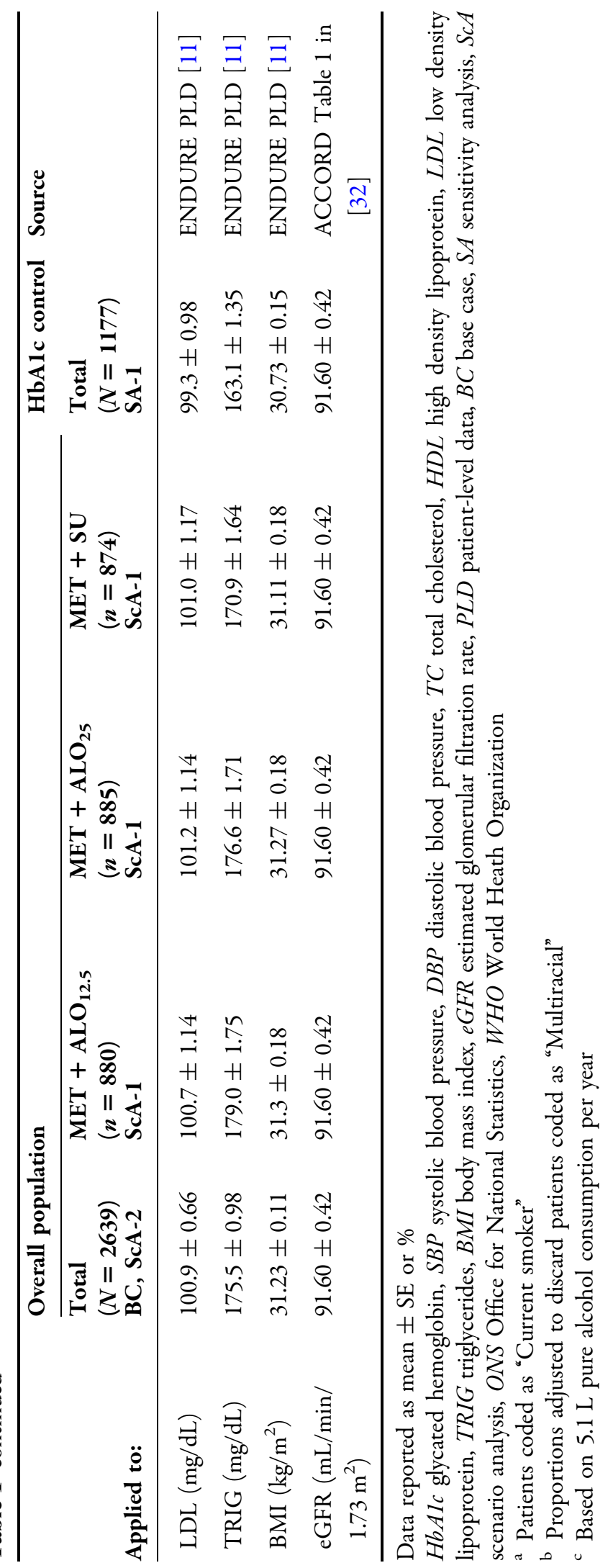




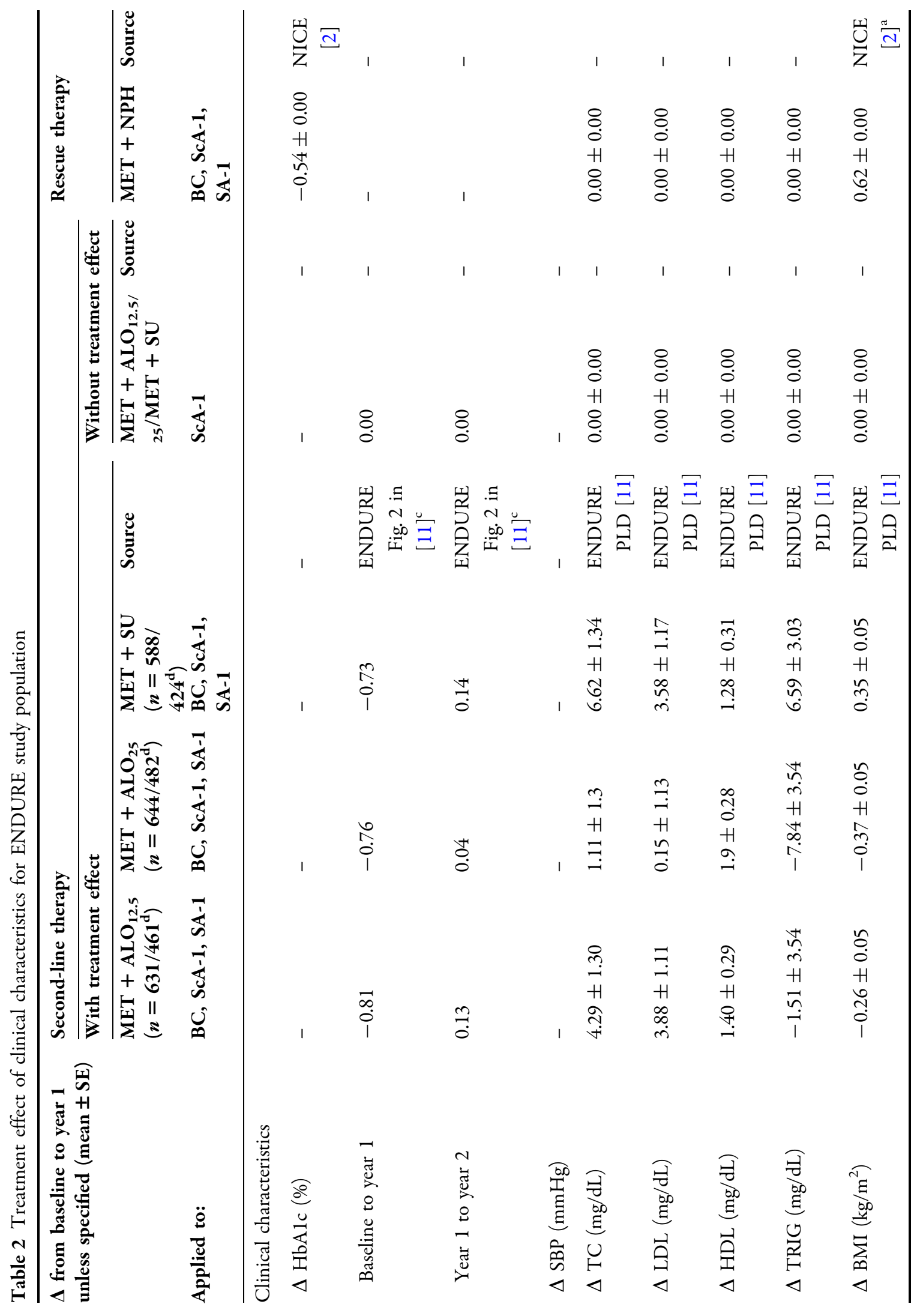




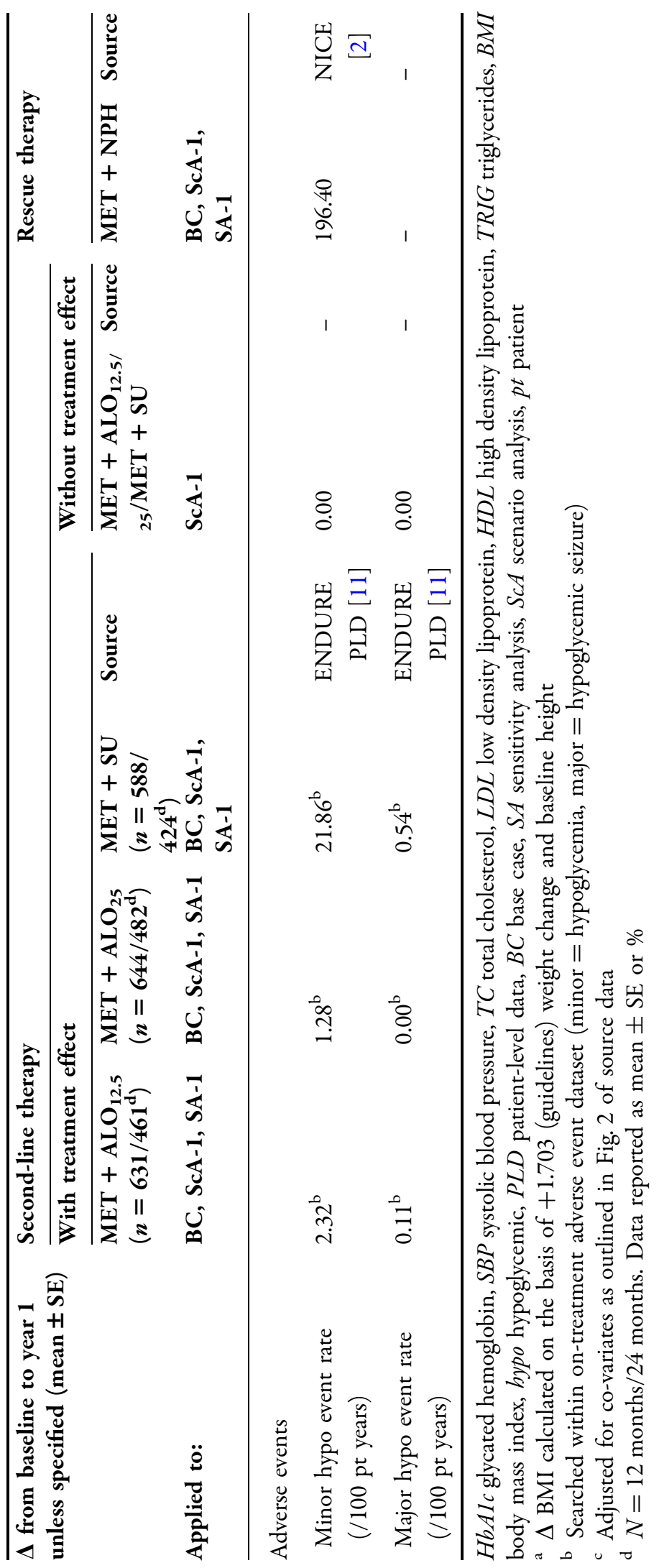




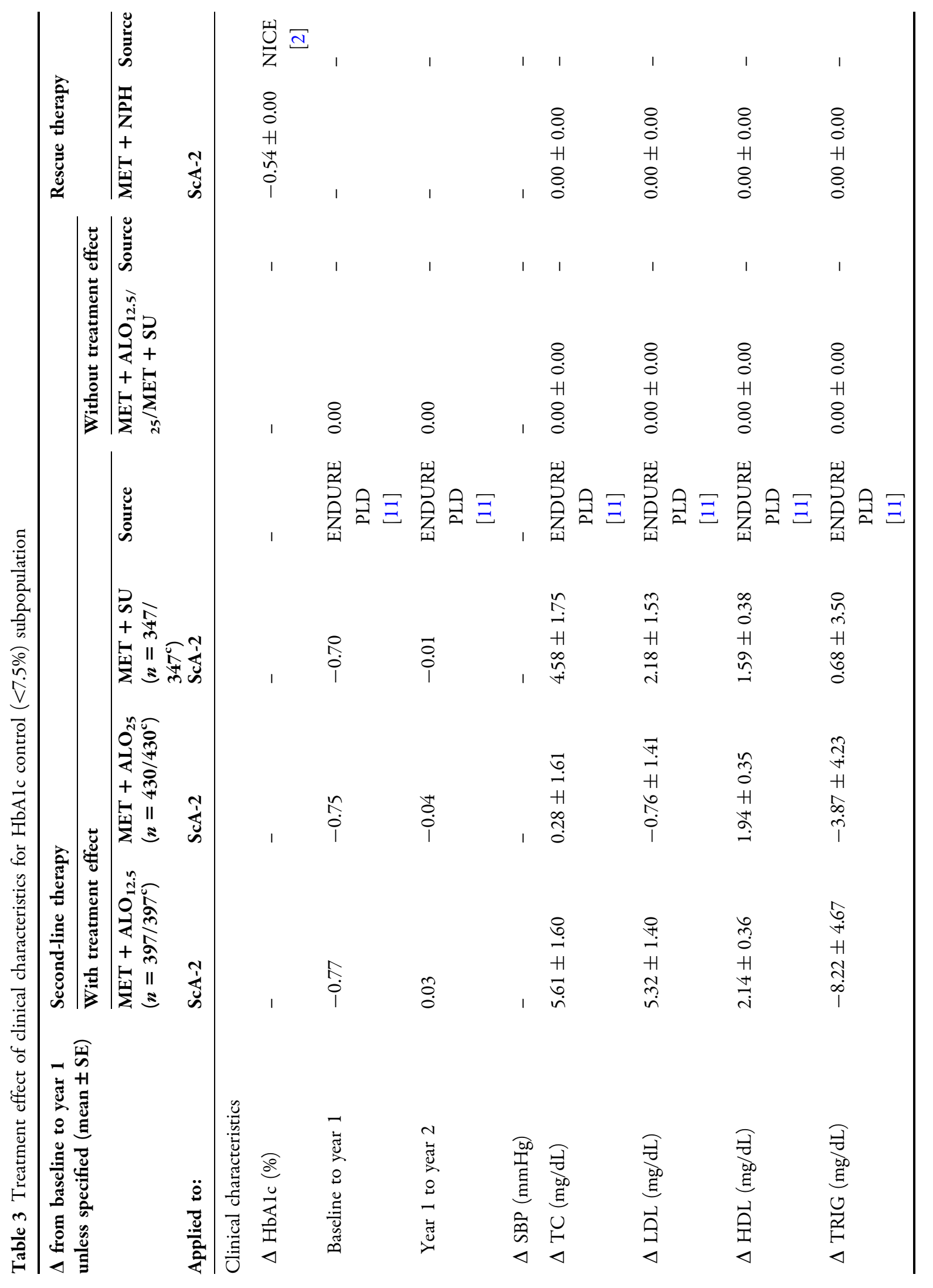




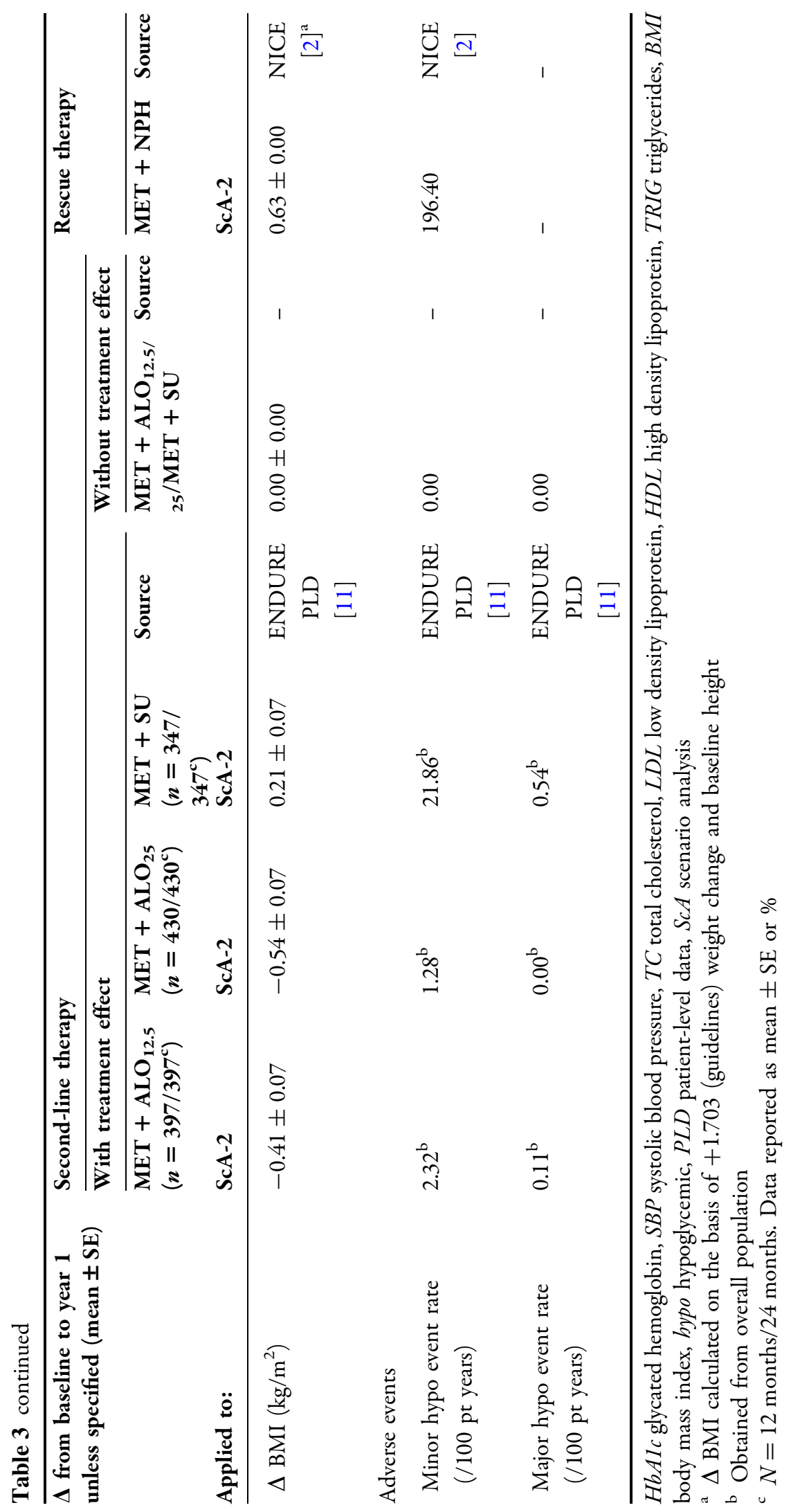




\section{Base Case Analyses}

The base case analysis considered the combined use of metformin and alogliptin $\left(\mathrm{MET}+\mathrm{ALO}_{12.5 / 25}\right)$ in patients with inadequately controlled $\mathrm{T} 2 \mathrm{DM}$, as an alternative to metformin and glipizide $(\mathrm{MET}+\mathrm{SU})$. In line with $\mathrm{UK}$ guidelines, therapy intensification occurred when HbA1c reached 7.5\%; at this point patients were escalated to insulin therapy: metformin and neutral protamine Hagedorn insulin $(\mathrm{MET}+\mathrm{NPH})$ [2].

\section{Probabilistic and Deterministic Sensitivity Analyses}

Additional analyses were performed for the base case including both probabilistic and a deterministic sensitivity analysis. For the probabilistic sensitivity analysis, 1000 runs were performed in which input parameters were sampled using the CDM's default distribution; for the deterministic sensitivity analysis, model inputs were fluctuated (10and 20-year time horizons; complication costs $\pm 20 \%$; utilities $\pm 20 \%$; discount rates (costs/ utilities) $0 \%$ and 7\%; duration switch of 5 years).

\section{Scenario Analyses (ScA)}

\section{ScA-1}

Scenario analyses assessed within-group comparisons using treatment arm-specific baseline profiles: MET + SU with no treatment effect versus $\mathrm{MET}+\mathrm{SU}$ with treatment effect; $\mathrm{MET}+\mathrm{ALO}_{12.5}$ with no treatment effect versus $\mathrm{MET}+\mathrm{ALO}_{12.5}$ with treatment effect; $\mathrm{MET}+\mathrm{ALO}_{25}$ with no treatment effect versus $\mathrm{MET}+\mathrm{ALO}_{25}$ with treatment effect.

\section{ScA-2}

A secondary scenario analysis replicated the base case simulations using a subpopulation of patients who achieved an HbA1c of $7.5 \%$ or less at 2 years, in line with NICE guidelines [2].

\section{Compliance with Ethics Guidelines}

This study was based on a previously conducted trial, and does not involve any new studies of human or animal subjects performed by any of the authors.

\section{RESULTS}

\section{Base Case Analyses}

The base case economic evaluation compared alogliptin (12.5 and $25 \mathrm{mg}$ doses) to $\mathrm{SU}$, as add-on therapies to metformin (Table 4, Fig. 1).

Treatment with alogliptin $12.5 \mathrm{mg}$ was estimated to incur additional total costs $(£ 1131)$ but gains in quality-adjusted life years (0.103 QALYs) and life expectancy (0.044 years). The additional total costs were driven by increased drug acquisition costs (£1399), which were partly offset by a reduction in complication costs (£263) from fewer predicted events. The largest cost offset in the analysis was attributable to a reduction in the incidence of CVD. Treatment with alogliptin $12.5 \mathrm{mg}$ compared with SU was associated with an incremental cost-effectiveness ratio (ICER) of $£ 10,959 /$ QALY.

Treatment with alogliptin $25 \mathrm{mg}$ was estimated to incur additional total costs (£1012) but gains in QALYs (0.140) and life expectancy (0.081 years). The additional total costs were driven by increased drug acquisition costs (£1421), which were partly offset by a reduction in complication costs $(£ 382)$ from 
Table 4 Base case event rate and economic analysis of alogliptin as a second-line antidiabetic therapy

\begin{tabular}{|c|c|c|c|}
\hline & $\mathbf{M E T}+\mathbf{S U}$ & $\mathrm{MET}+\mathrm{ALO}_{12.5}$ & $\mathrm{MET}+\mathrm{ALO}_{25}$ \\
\hline \multicolumn{4}{|c|}{ Macrovascular complications (cumulative incidence \%) } \\
\hline CHF death & 39.48 & 40.46 & 40.80 \\
\hline CHF event & 15.72 & 15.23 & 15.19 \\
\hline PVD onset & 19.26 & 19.07 & 18.79 \\
\hline Angina & 13.72 & 13.39 & 13.06 \\
\hline Diabetes mortality & 26.97 & 26.73 & 26.81 \\
\hline Stroke event & 7.66 & 7.64 & 7.53 \\
\hline Event fatality & 33.36 & 32.64 & 32.21 \\
\hline MI event & 18.42 & 17.85 & 17.63 \\
\hline \multicolumn{4}{|c|}{ Microvascular complications (cumulative incidence \%) } \\
\hline Background diabetic retinopathy & 29.62 & 29.29 & 29.35 \\
\hline Proliferative diabetic retinopathy & 2.56 & 2.49 & 2.48 \\
\hline Macular edema & 25.47 & 25.14 & 25.19 \\
\hline Severe vision loss & 12.83 & 12.55 & 12.56 \\
\hline Cataract & 13.09 & 13.05 & 13.08 \\
\hline Microalbuminuria & 41.25 & 41.00 & 40.88 \\
\hline Gross proteinuria & 14.80 & 14.59 & 14.48 \\
\hline ESRD & 4.86 & 4.78 & 4.66 \\
\hline Nephropathy (death) & 0.00 & 0.00 & 0.00 \\
\hline Ulcer & 41.9 & 41.52 & 41.57 \\
\hline Recurrent ulcer & 89.6 & 88.72 & 88.88 \\
\hline Amputation due to ulcer & 19.53 & 19.34 & 19.43 \\
\hline Amputation due to recurrent ulcer & 13.41 & 13.30 & 13.38 \\
\hline Neuropathy & 72.8 & 72.53 & 72.49 \\
\hline \multicolumn{4}{|l|}{ Absolute results (discounted) } \\
\hline Total cost $(£)$ & 27,835 & 28,966 & 28,847 \\
\hline Treatment & 6644 & 8043 & 8065 \\
\hline Management & 462 & 463 & 465 \\
\hline CVD & 7450 & 7358 & 7259 \\
\hline ESRD & 1245 & 1186 & 1164 \\
\hline Ulcer/amputation/neuropathy & 10,130 & 10,038 & 10,043 \\
\hline Eye & 1851 & 1831 & 1828 \\
\hline
\end{tabular}


Table 4 continued

\begin{tabular}{|c|c|c|c|}
\hline & MET + SU & $\mathrm{MET}+\mathrm{ALO}_{12.5}$ & $\mathrm{MET}+\mathrm{ALO}_{25}$ \\
\hline Hypoglycemia & 0 & 0 & 0 \\
\hline Total LE & 14.833 & 14.878 & 14.914 \\
\hline Total QALY & 9.720 & 9.824 & 9.861 \\
\hline \multicolumn{4}{|c|}{ Between groups analysis $\left(\mathrm{MET}+\mathrm{SU}\right.$ vs $\left.\mathrm{MET}+\mathrm{ALO}_{12.5 / 25}\right)$} \\
\hline Incremental cost & & 1131 & 1012 \\
\hline Incremental LE & & 0.044 & 0.081 \\
\hline Incremental QALY & & 0.103 & 0.14 \\
\hline ICER (cost/LE) & & 25,588 & 12,476 \\
\hline ICER (cost/QALY) & & 10,959 & 7217 \\
\hline CE? ( $£ 30,000$ ICER $)$ & & 67.6 & 77.1 \\
\hline
\end{tabular}

$M I$ myocardial infarction, $C V D$ cardiovascular disease, ESRD end-stage renal disease, $L E$ life expectancy, $Q A L Y$ quality-adjusted life year, ICER incremental cost-effectiveness ratio, $C E$ ? probability of cost-effectiveness

fewer predicted events. The largest cost offset in the analysis was attributable to a reduction in the incidence of CVD. Treatment with alogliptin $25 \mathrm{mg}$ compared with SU was associated with an ICER of $£ 7217 /$ QALY.

Results of the probabilistic sensitivity analysis support the base case results and give an indication as to the likelihood of cost-effectiveness at various willingness to pay thresholds. ICER scatterplots (Figs. 2, 3) demonstrate that in the comparison of alogliptin $12.5 \mathrm{mg}$ and $\mathrm{SU}$, alogliptin $12.5 \mathrm{mg}$ was cost-effective at a threshold of $£ 30,000 /$ QALY with a probability of cost-effectiveness of $67.6 \%$. Similarly, in the analysis of alogliptin $25 \mathrm{mg}$ and SU, the probability of cost-effectiveness of alogliptin $25 \mathrm{mg}$ was $77.1 \%$ at a $£ 30,000 / \mathrm{QALY}$ willingness to pay threshold.

Results of the deterministic sensitivity analysis are reported in Table 5. The cost-effectiveness of alogliptin 12.5 and $25 \mathrm{mg}$ was insensitive to change in key model input parameters and remained cost-effective compared to SU across deterministic sensitivity analyses. For alogliptin $12.5 \mathrm{mg}$, ICERs across sensitivity analyses ranged from $£ 6932 / \mathrm{QALY}$ to $£ 24,143 /$ QALY (base case ICER $£ 10,959 / \mathrm{QALY}$ ). For alogliptin $25 \mathrm{mg}$, ICERs across sensitivity analyses ranged from $£ 4225 / \mathrm{QALY}$ to $£ 19,056$ / QALY (base case ICER £7217/QALY). ICERs improved with increased time horizon driven by increased accumulation of QALYs. However, even at a 10-year time horizon, alogliptin was cost-effective compared with SU with ICERs less than $£ 20,000 / Q A L Y$ at 12.5 and $25 \mathrm{mg}$ doses.

\section{Scenario Analysis (ScA)}

\section{ScA-1}

A within-arm cost-effectiveness analysis was undertaken for each treatment group: SU, alogliptin $12.5 \mathrm{mg}$, and alogliptin $25 \mathrm{mg}$. In each analysis, patient baseline profiles were 


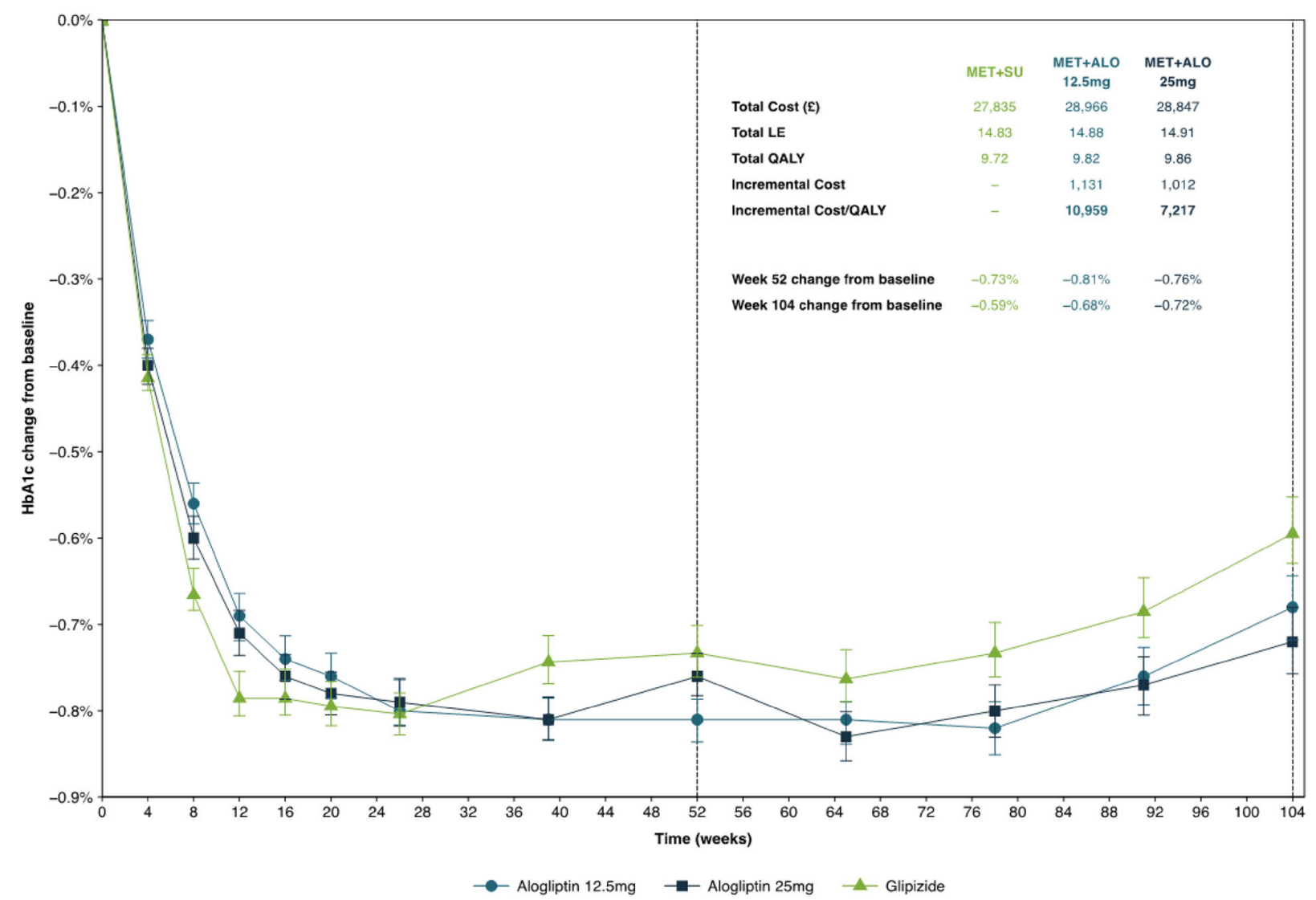

Fig. 1 Relationship between sustained antihyperglycemic efficacy (HbAlc) and cost-effectiveness of alogliptin $12.5 \mathrm{mg}$ and $25 \mathrm{mg}$ vs SU ([adapted from [11])

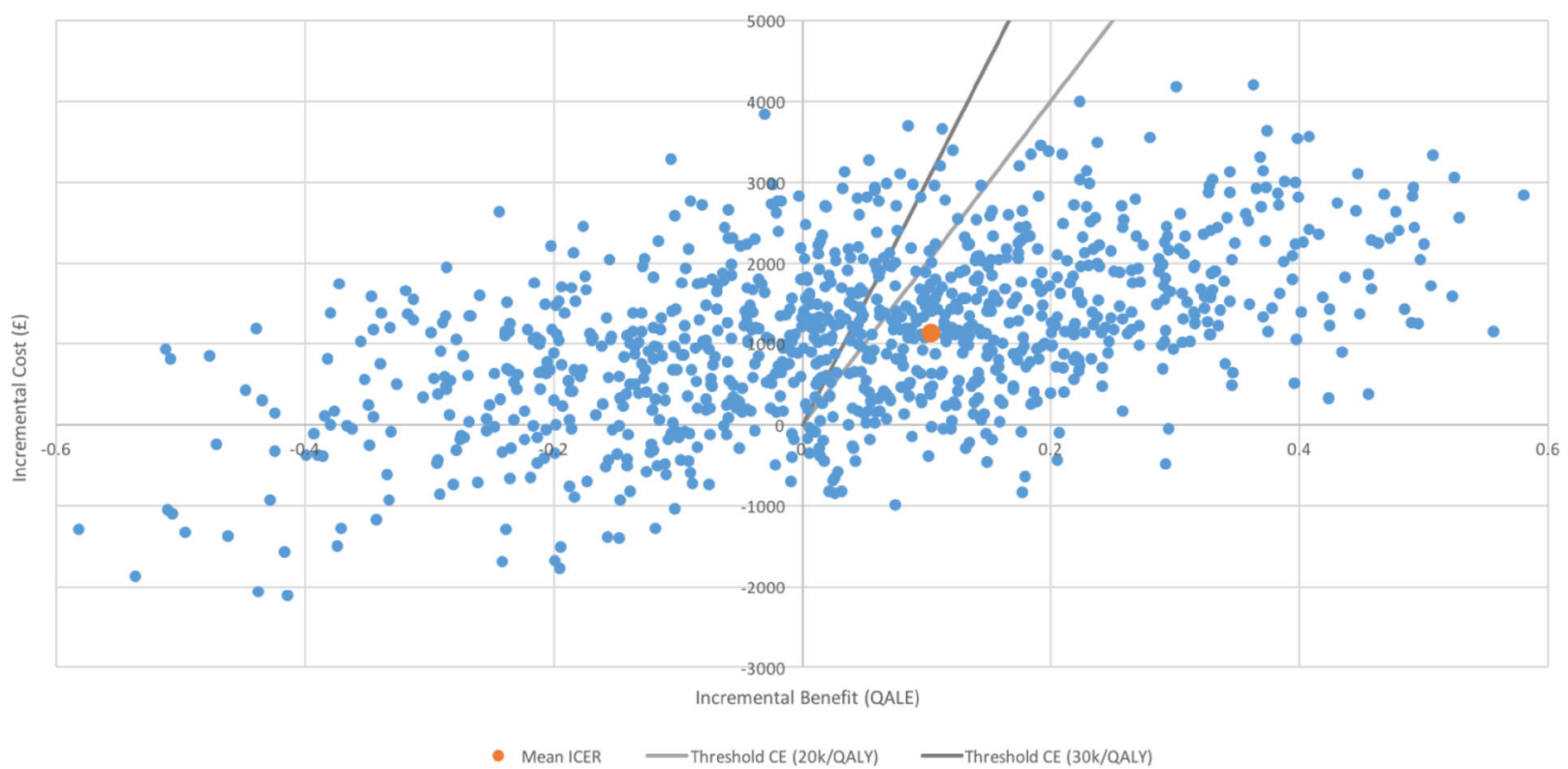

Fig. 2 Incremental cost-effectiveness ratio scatterplot (SU vs alogliptin $12.5 \mathrm{mg}$ ) 


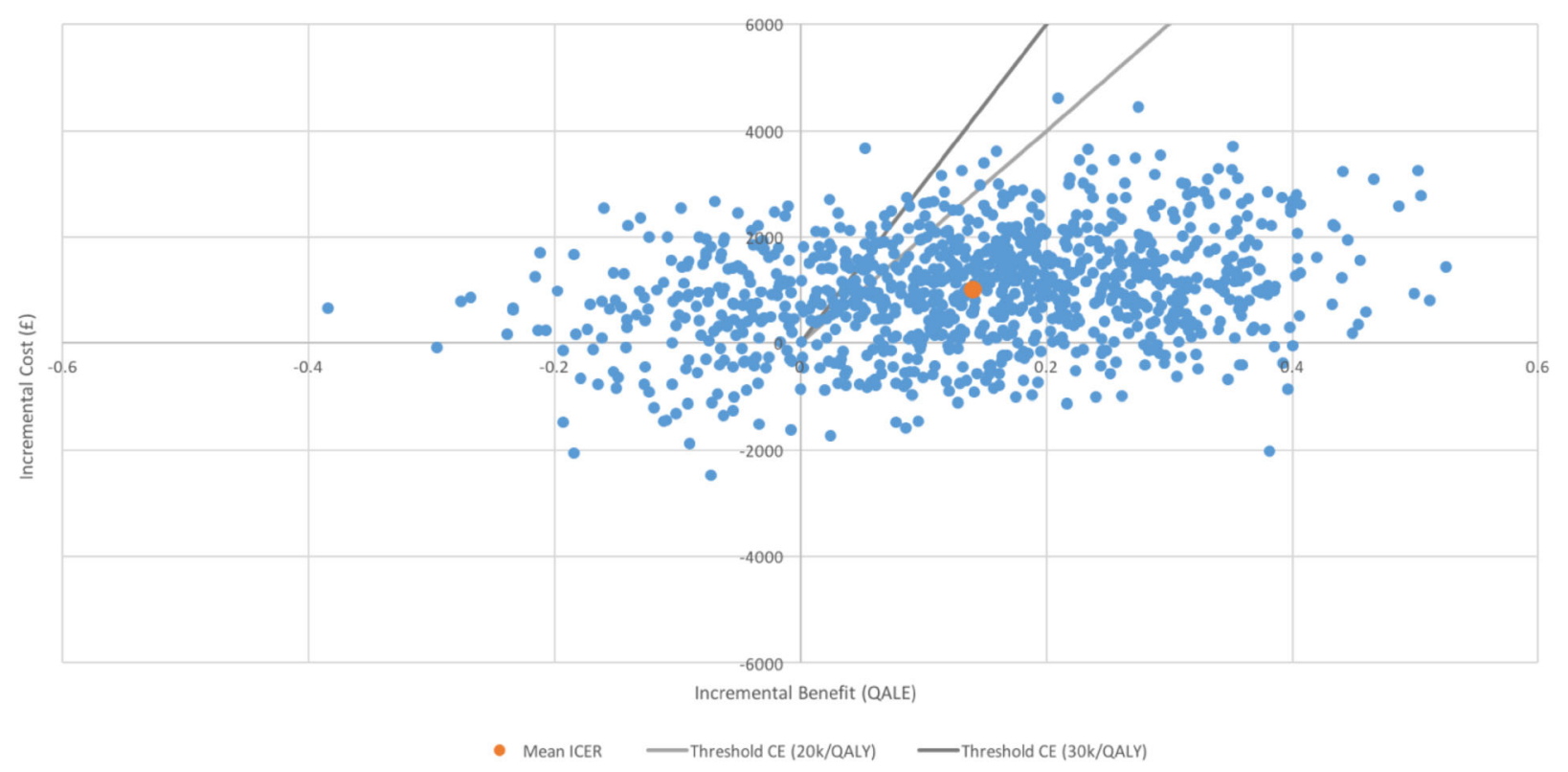

Fig. 3 Incremental cost-effectiveness ratio scatterplot (SU vs alogliptin $25 \mathrm{mg}$ )

compared to 12-month profiles (12 and 24 months for $\mathrm{HbA1c}$ ), to evaluate the cost-effectiveness of each treatment allocation.

Treatment with SU was estimated to incur higher total costs $(£ 2194)$ but gains in QALYs (0.211) and life expectancy (0.291 years) as an add-on to metformin. The higher total costs were driven by an increase in drug acquisition costs, but partly offset by a corresponding decrease in complication costs from fewer predicted events. The largest cost components in the analysis were attributable to the incidence of ulcer, amputation, and neuropathy. The addition of an SU to metformin was associated with an ICER of $£ 10,398 /$ QALY (Table 6).

Treatment with alogliptin $12.5 \mathrm{mg}$ was estimated to incur additional total costs (£3325) but gains in QALYs (0.315) and life expectancy ( 0.336 years). The additional total costs were driven by increased drug acquisition costs, which were partly offset by a reduction in complication costs from fewer predicted events.
The largest cost offsets in the analysis were attributable to CVD and renal disease, and the incidence of ulcer amputation and neuropathy. The addition of alogliptin $12.5 \mathrm{mg}$ to metformin was associated with an ICER of $£ 10,556 /$ QALY (Table 6).

Treatment with alogliptin $25 \mathrm{mg}$ was estimated to incur additional total costs ( $£ 3206)$ but gains in QALYs (0.352) and life expectancy (0.372 years). The additional total costs were driven by increased drug acquisition costs, which were partly offset by a reduction in complication costs from fewer predicted events. The largest cost offsets in the analysis were attributable to CVD and renal disease, and the incidence of ulcer amputation and neuropathy. The addition of alogliptin $25 \mathrm{mg}$ to metformin was associated with an ICER of $£ 9108 /$ QALY (Table 6).

\section{ScA-2}

A subgroup economic evaluation was undertaken of the base case population (between-arm comparison of SU and 
Table 5 Deterministic sensitivity analysis results (SU vs alogliptin $12.5 \mathrm{mg}$ and $25 \mathrm{mg}$ )

\begin{tabular}{|c|c|c|c|}
\hline Strategy vs MET + SU & $\begin{array}{l}\text { Incremental } \\
\text { cost }(\boldsymbol{E})\end{array}$ & $\begin{array}{l}\text { Incremental } \\
\text { benefit (QALY) }\end{array}$ & $\begin{array}{l}\text { Incremental cost-effectiveness } \\
(\mathfrak{E} / \mathrm{QALY})\end{array}$ \\
\hline \multicolumn{4}{|l|}{$\mathrm{MET}+\mathrm{ALO}_{12.5 \mathrm{mg}}$} \\
\hline Base case & 1131 & 0.103 & 10,959 \\
\hline 10-year horizon & 1297 & 0.054 & 24,143 \\
\hline 20-year horizon & 1109 & 0.082 & 13,571 \\
\hline Costs $-20 \%$ & 1184 & 0.103 & 11,477 \\
\hline Utilities - $20 \%$ & 1131 & 0.094 & 11,993 \\
\hline Costs $+20 \%$ & 1078 & 0.103 & 10,441 \\
\hline Utilities $+20 \%$ & 1131 & 0.112 & 10,098 \\
\hline Discount rate $0 \%$ & 1121 & 0.162 & 6932 \\
\hline Discount rate $7 \%$ & 1074 & 0.072 & 14,961 \\
\hline Duration switch 5 years & 1008 & 0.082 & 12,252 \\
\hline \multicolumn{4}{|l|}{$\mathrm{MET}+\mathrm{ALO}_{25} \mathrm{mg}$} \\
\hline Base case & 1012 & 0.140 & 7217 \\
\hline 10-year horizon & 1201 & 0.063 & 19,056 \\
\hline 20-year horizon & 1000 & 0.109 & 9200 \\
\hline Costs $-20 \%$ & 1093 & 0.140 & 7799 \\
\hline Utilities $-20 \%$ & 1012 & 0.125 & 8101 \\
\hline Costs $+20 \%$ & 930 & 0.140 & 6635 \\
\hline Utilities $+20 \%$ & 1012 & 0.155 & 6515 \\
\hline Discount rate $0 \%$ & 1022 & 0.242 & 4225 \\
\hline Discount rate $7 \%$ & 978 & 0.091 & 10,721 \\
\hline Duration switch 5 years & 877 & 0.120 & 7306 \\
\hline
\end{tabular}

alogliptin), to assess the cost-effectiveness profile of subjects who maintained a level of HbA1c at 2 years (104 weeks) of less than $7.5 \%$. Results of this scenario analysis were similar to the base case analysis in terms of absolute costs and health benefits, with ICERs (probability of cost-effectiveness at $£ 30,000 / \mathrm{QALY})$ of $£ 13,326$ / QALY (61.0\%) and $£ 6771 /$ QALY (72.4\%) for the comparison of SU and alogliptin $12.5 \mathrm{mg}$ and $25 \mathrm{mg}$, respectively (Table 7).

\section{DISCUSSION}

As a result of both the incidence and increasing prevalence of T2DM in the UK, the consequences of suboptimal glycemic control impose a considerable economic burden on patients and the NHS. These costs are further exacerbated when current treatment strategies lack the glycemic durability required to manage the progressive nature of the condition. When 
Table 6 Intragroup economic analysis of SU, alogliptin $12.5 \mathrm{mg}$, and alogliptin $25 \mathrm{mg}$ as a second-line antidiabetic therapy

\begin{tabular}{|c|c|c|c|c|c|c|}
\hline & \multicolumn{2}{|c|}{$\mathbf{M E T}+\mathbf{S U}$} & \multicolumn{2}{|c|}{$\mathrm{MET}+\mathrm{ALO}_{12.5}$} & \multicolumn{2}{|c|}{$\mathrm{MET}+\mathrm{ALO}_{25}$} \\
\hline & Baseline & Month 12 & Baseline & Month 12 & Baseline & Month 12 \\
\hline \multicolumn{7}{|c|}{ Absolute results (discounted) } \\
\hline Total cost $(£)$ & 25,641 & 27,835 & 25,641 & 28,966 & 25,641 & 28,847 \\
\hline Total LE & 14.542 & 14.833 & 14.542 & 14.878 & 14.542 & 14.914 \\
\hline Total QALY & 9.509 & 9.720 & 9.509 & 9.824 & 9.509 & 9.861 \\
\hline Incremental cost & & 2194 & & 3325 & & 3206 \\
\hline Incremental LE & & 0.291 & & 0.336 & & 0.372 \\
\hline Incremental QALY & & 0.211 & & 0.315 & & 0.352 \\
\hline ICER (cost/LE) & & 7540 & & 9896 & & 8618 \\
\hline ICER (cost/QALY) & & 10,398 & & 10,556 & & 9108 \\
\hline
\end{tabular}

$L E$ life expectancy, $Q A L Y$ quality-adjusted life year, ICER incremental cost-effectiveness ratio

Table 7 Economic analysis of SU, alogliptin $12.5 \mathrm{mg}$, and alogliptin $25 \mathrm{mg}$ for HbAlc control (<7.5\%) subpopulation

\begin{tabular}{|c|c|c|c|}
\hline & $\mathrm{MET}+\mathrm{SU}$ & $\mathrm{MET}+\mathrm{ALO}_{12.5}$ & $\mathrm{MET}+\mathrm{ALO}_{25}$ \\
\hline \multicolumn{4}{|c|}{ Absolute results (discounted) } \\
\hline Total cost $(£)$ & 28,688 & 29,565 & 29,435 \\
\hline Total LE & 14.641 & 14.663 & 14.708 \\
\hline Total QALY & 9.603 & 9.688 & 9.713 \\
\hline \multicolumn{4}{|c|}{ Between groups analysis $\left(\mathrm{MET}+\mathrm{SU}\right.$ vs $\left.\mathrm{MET}+\mathrm{ALO}_{12.5 / 25}\right)$} \\
\hline Incremental cost & & 877 & 746 \\
\hline Incremental LE & & 0.022 & 0.068 \\
\hline Incremental QALY & & 0.066 & 0.110 \\
\hline ICER (cost/LE) & & 39,856 & 11,039 \\
\hline ICER (cost/QALY) & & 13,326 & 6771 \\
\hline CE? (£30,000 ICER) & & 61.0 & 72.4 \\
\hline
\end{tabular}

LE life expectancy, $Q A L Y$ quality-adjusted life year, ICER incremental cost-effectiveness ratio, CE? probability of cost-effectiveness

compared to standard second-line SU therapy, the ENDURE trial showed that DPP-4 inhibition by alogliptin elicited sustained antihyperglycemic effects, without weight gain and hypoglycemia, in T2DM patients who had failed first-line metformin monotherapy $[11,12]$.

The present economic analysis of ENDURE aimed to quantify the relationship between improved glycemic durability and 
cost-effectiveness of alogliptin in the UK clinical setting. This study estimated that in patients inadequately managed on metformin monotherapy, the addition of alogliptin (12.5 and $25 \mathrm{mg}$ ) was associated with improvements in length and quality of life and was cost-effective at established norms. Compared with baseline profiles of suboptimal management on metformin monotherapy (intragroup analysis), combination therapies with alogliptin or SU were associated with improvements in length and quality of life and were cost-effective at established norms. However, the increased glycemic durability associated with alogliptin translated to larger health (QALY) gains predicted across analyses compared with SU.

Improvements in glycemic control and durability of antihyperglycemic effects observed in ENDURE subjects translates to favorable cost-effectiveness profiles for alogliptin compared with $\mathrm{SU}$, as add-on therapies to metformin when analyzed with an established diabetes model. These cost-effectiveness conclusions were robust across a number of scenarios, including intragroup analyses that confirmed the cost-effectiveness of the addition of SU and alogliptin in analyses where patients within each study arm formed their own control group. In patients who had HbA1c levels less than $7.5 \%$ after 2 years, alogliptin was estimated to be cost-effective compared with $\mathrm{SU}$ at established norms. In deterministic and probabilistic sensitivity analyses where the joint uncertainty in parameter values was evaluated in terms of its impact on estimates of cost-effectiveness, base case cost-effectiveness conclusions were shown to be robust. In comparison with $\mathrm{SU}$, the probability that combination therapy with alogliptin (12.5 and $25 \mathrm{mg}$ ) was cost-effective was $67.6 \%$ and $77.1 \%$ at a willingness to pay threshold of $£ 30,000$ per QALY gained, respectively.

This economic evaluation of ENDURE provides further evidence supporting the cost-effectiveness of DPP-4 inhibitors as a second-line therapy for T2DM [20]. Previous analyses have indicated that, in T2DM patients who were no longer responsive to first-line metformin monotherapy, the addition of DPP-4 inhibitors was cost-effective compared to add-on SU [21-25], thiazolidinediones $[25,26]$, and insulin $[27,28]$. The DPP-4 inhibitors investigated in these studies were either sitagliptin or saxagliptin; however, a pharmacoeconomic analysis of antidiabetic therapies in the Japanese clinical setting found that alogliptin was a more cost-effective DPP-4 inhibitor than sitagliptin [29]. The ENDURE trial and its subsequent cost-effectiveness analysis suggest that the improved efficacy of second-line alogliptin therapy translated to improved cost-effectiveness compared to SU in patients with uncontrolled T2DM.

There are several strengths and limitations associated with this study. A UK perspective was adopted for costs and cost-effectiveness settings (e.g., discount rates), which may affect whether these findings are relatable to other country settings. However, the input profiles and treatment effects from ENDURE were based on subjects from North and South America, Europe, Asia, South Africa, Australia, and New Zealand [11] and are reported transparently such that country-specific settings for costs and utilities could be used to replicate this analysis to inform country-specific decision-making. Computer modelling in diabetes is an established and accepted paradigm, and is used to extrapolate beyond the trial follow-up period to obtain best estimates of downstream clinical and economic outcomes associated with individual 
treatments. Nonetheless, a computer simulation model was used to evaluate how changes in subjects' short-term surrogate outcomes (risk factor profiles) translated to incidence of diabetes-related complications and mortality over a lifetime perspective. Given the lifetime nature of the analysis, assumptions regarding patient treatment escalation were made such that patients escalated (or intensified) to rescue therapy once their HbA1c value (following initial treatment-related change) returned to its starting (or baseline) HbA1c. This is a realistic assumption that may reflect treatment intensification practice in the clinical setting. In the base case analysis, the SU and alogliptin arms intensified to metformin and NPH insulin after 7-9 years across analyses; in the modelled lifetime analysis, discounted average life expectancy was approximately 14-15 years. Therefore, the comparison of alogliptin and SU contains the effects of therapy intensification for the period of the modelled time horizon, which should be acknowledged when interpreting the results. However, as the therapy intensification profile was applied equally to each arm, any incremental differences associated with therapy escalations should pertain to different times to escalation which were not substantially different.

\section{CONCLUSION}

The use of SU as a second-line indication for uncontrolled T2DM is associated with weight gain and hypoglycemic events [7], the risks of which are further increased when doses are escalated to combat progressive treatment failure [6]. In comparison, the ENDURE trial showed that alogliptin, in combination with metformin, was associated with improved glycemic durability, without weight gain and hypoglycemia, over 2 years $[11,12]$. With base case estimated ICERs of $£ 10,959 /$ QALY and $£ 7217 / \mathrm{QALY}$, evaluated against commonly used cost-effectiveness threshold values, this study demonstrated that alogliptin represents a cost-effective treatment alternative to $\mathrm{SU}$ as add-on therapy to metformin in patients with poorly managed T2DM. Cost-effectiveness conclusions were robust across a number of evaluated scenarios and in sensitivity analyses.

\section{ACKNOWLEDGEMENTS}

Sponsorship for this study and article processing charges were funded by Takeda Development Centre Europe Ltd. All named authors meet the International Committee of Medical Journal Editors (ICMJE) criteria for authorship for this manuscript, take responsibility for the integrity of the work as a whole, and have given final approval to the version to be published. Editorial assistance in the preparation of this manuscript was provided by Dr Karina Hamilton of HEOR Ltd. Support for this assistance was funded by HEOR Ltd.

J. Gordon and P. McEwan were involved in the design, analysis, interpretation, and writing of the manuscript. J. Puelles was involved in the design, interpretation, and writing of the manuscript. M. Hurst was involved in the design and analysis of the manuscript.

All authors had full access to all of the data in this study and take complete responsibility for the integrity of the data and accuracy of the data analysis.

Disclosures. J. Puelles is an employee of Takeda Development Centre Europe Ltd. J. Gordon has served as consultant to and received research funding from Takeda 
Development Centre Europe Ltd in relation to this study. P. McEwan has served as consultant to and received research funding from Takeda Development Centre Europe Ltd in relation to this study. M. Hurst has served as consultant to and received research funding from Takeda Development Centre Europe Ltd in relation to this study.

Compliance with Ethics Guidelines. This article is based on previously conducted studies, and does not involve any new studies of human or animal subjects performed by any of the authors.

Data Availability. The datasets analyzed during the current study were sourced from and are available in the original trial publication [11].

Open Access. This article is distributed under the terms of the Creative Commons Attribution-NonCommercial 4.0 International License (http://creativecommons.org/licenses/ by-nc/4.0/), which permits any noncommercial use, distribution, and reproduction in any medium, provided you give appropriate credit to the original author(s) and the source, provide a link to the Creative Commons license, and indicate if changes were made.

\section{REFERENCES}

1. Hex N, Bartlett C, Wright D, Taylor M, Varley D. Estimating the current and future costs of type 1 and type 2 diabetes in the UK, including direct health costs and indirect societal and productivity costs. Diabet Med. 2012;29:855-62.

2. National Institute for Health and Care Excellence. Type 2 diabetes in adults: management. NICE guidelines [NG28]. 2015. https://www.nice.org.uk/ guidance/ng28. Accessed 22 Mar 2016.
3. National Diabetes Audit. National Diabetes Audit 2011-2012. Report 1: Care processes and treatment targets. 2013. http://www.hscic.gov.uk/catalogue/ PUB12421/nati-diab-audi-11-12-care-proc-rep.pdf. Accessed 22 Mar 2016.

4. American Diabetes Association. Standards of Medical Care in Diabetes-2016. Diabetes Care. 2016;39(S1):S1-112.

5. Turner RC, Cull CA, Frighi V, Holman RR. Glycemic control with diet, sulfonylurea, metformin, or insulin in patients with type 2 diabetes mellitus: progressive requirement for multiple therapies (UKPDS49). JAMA. 1999;281(21):2005-12.

6. Kahn SE, Haffner SM, Heise MA, et al. Glycemic durability of rosiglitazone, metformin, or glyburide monotherapy. $\mathrm{N}$ Engl J Med. 2006;355(23):2427-43.

7. Phung OJ, Scholle JM, Talwar M, Coleman CI. Effect of noninsulin antidiabetic drugs added to metformin therapy on glycemic control, weight gain, and hypoglycemia in type 2 diabetes. JAMA. 2010;303(14):1410-8.

8. DeFronzo RA, Fleck PR, Wilson CA, Mekki Q. Efficacy and safety of the dipeptidyl peptidase-4 inhibitor alogliptin in patients with type 2 diabetes and inadequate glycemic control: a randomized, double-blind, placebo-controlled study. Diabetes Care. 2008;31:2315-7.

9. Nauck MA, Ellis GC, Fleck PR, Wilson CA, Mekki Q. Efficacy and safety of adding the dipeptidyl peptidase-4 inhibitor alogliptin to metformin therapy in patients with type 2 diabetes inadequately controlled with metformin monotherapy: a multicentre, randomised, double-blind, placebo-controlled study. Int J Clin Pract. 2009;63:46-55.

10. Bosi E, Ellis GC, Wilson CA, Fleck PR. Alogliptin as a third oral antidiabetic drug in patients with type 2 diabetes and inadequate glycaemic control on metformin and pioglitazone: a 52-week, randomized, double-blind, active-controlled, parallel-group study. Diabetes Obes Metab. 2011;13:1088-96.

11. Del Prato S, Camisasca R, Wilson C, Fleck P. Durability of the efficacy and safety of alogliptin compared with glipizide in type 2 diabetes mellitus: a 2-year study. Diabetes Obes Metab. 2014;16:1239-46.

12. Del Prato S, Fleck P, Wilson C, Chaudhari P. Comparison of alogliptin and glipizide for composite endpoint of glycated haemoglobin reduction, no hypoglycaemia and no weight gain 
in type 2 diabetes mellitus. Diabetes Obes Metab. 2016;18(6):623-7.

13. Palmer AJ, Roze S, Valentine WJ, et al. The CORE Diabetes Model: projecting long-term clinical outcomes, costs and costeffectiveness of interventions in diabetes mellitus (types 1 and 2) to support clinical and reimbursement decision-making. Curr Med Res Opin. 2004;20(Suppl 1):S5-26.

14. Diabetes Control and Complications Trial Research Group. The effect of intensive treatment of diabetes on the development and progression of long-term complications in insulin-dependent diabetes mellitus. N Engl J Med. 1993;329(14):977-86.

15. D'Agostino RB, Russell MW, Huse DM, et al. Primary and subsequent coronary risk appraisal: new results from the Framingham study. Am Heart J. 2000;139(2):272-81.

16. Alva ML, Gray A, Mihaylova B, Leal J, Holman R. The impact of diabetes-related complications on healthcare costs: new results from the UKPDS (UKPDS 84). Diabet Med. 2015;32(4):459-66.

17. Clarke P, Gray A, Holman R. Estimating utility values for health states of type 2 diabetic patients using the EQ-5D (UKPDS 62). Med Decis Mak. 2002;22(4):340-9.

18. Personal Social Services Research Unit. Unit costs of health and social care 2015. 2015. http://www. pssru.ac.uk/project-pages/unit-costs/2015/index.php. Accessed 23 Mar 2016.

19. Monthly Index of Medical Specialities. 2016. http:// www.mims.co.uk/. Accessed 23 Mar 2016.

20. Geng J, Yu H, Mao Y, Zhang P, Chen Y. Cost effectiveness of dipeptidyl peptidase- 4 inhibitors for type 2 diabetes. Pharmacoeconomics. 2015;33(6):581-97.

21. Bergenheim K, Williams SA, Bergeson JG, Stern L, Sriprasert M. US cost-effectiveness of saxagliptin in type 2 diabetes mellitus. Am J Pharm Benefits. 2012;4(1):20-8.

22. Elgart JF, Caporale JE, Gonzalez L, Aiello E, Waschbusch M, Gagliardino JJ. Treatment of type 2 diabetes with saxagliptin: a pharmacoeconomic evaluation in Argentina. Health Econ Rev. 2013;3(1):1-9.
23. Erhardt W, Bergenheim K, Duprat-Lomon I, McEwan P. Cost effectiveness of saxagliptin and metformin versus sulfonylurea and metformin in the treatment of type 2 diabetes mellitus in Germany. Clin Drug Investig. 2012;32(3):189-202.

24. Granström O, Bergenheim K, McEwan P, Sennfält $\mathrm{K}$, Henriksson M. Cost-effectiveness of saxagliptin $\left(\right.$ Onglyza $^{\circledR}$ ) in type 2 diabetes in Sweden. Prim Care Diabetes. 2012;6(2):127-36.

25. Schwarz B, Gouveia M, Chen J, et al. Cost-effectiveness of sitagliptin-based treatment regimens in European patients with type 2 diabetes and haemoglobin A1c above target on metformin monotherapy. Diabetes Obes Metab. 2008;10(Suppl 1):43-55.

26. Nita ME, Eliaschewitz FG, Ribeiro E, et al. Cost-effectiveness and budget impact of saxagliptine as additional therapy to metformin for the treatment of diabetes mellitus type 2 in the Brazilian private health system. Rev Assoc Med Bras. 2012;58(3):294-301.

27. Grzeszczak W, Czupryniak L, Kolasa K, Sciborski C, Lomon ID, McEwan P. The cost-effectiveness of saxagliptin versus NPH insulin when used in combination with other oral antidiabetes agents in the treatment of type 2 diabetes mellitus in Poland. Diabetes Technol Ther. 2012;14(1):65-73.

28. Klarenbach S, Cameron C, Singh S, Ur E. Cost-effectiveness of second-line antihyperglycemic therapy in patients with type 2 diabetes mellitus inadequately controlled on metformin. CMAJ. 2011;183(16):E1213-20.

29. Teramachi $H$, Ohta $H$, Tachi $T$, et al. Pharmacoeconomic analysis of DPP-4 inhibitors. Pharmazie. 2013;68(11):909-15.

30. Office for National Statistics. Adult smoking habits in Great Britain. London: Office for National Statistics; 2013.

31. World Health Organization. Global Status Report on Alcohol. Geneva: World Health Organization; 2004.

32. ACCORD Study Group. Effects of intensive blood-pressure control in type 2 diabetes mellitus. N Engl J Med. 2010;362(17):1575-85. 